\title{
Mobile Technologies in Healthcare: Approaches and Architecture
}

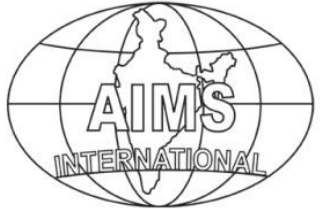

Volume 11, Number 1

January 2017, pp. 1-19

\author{
Mukta Mukherjee \\ Suresh Chalasani \\ Derek Riley \\ University of Wisconsin-Parkside \\ (mukhe001@rangers.uwp.edu) \\ (chalasan@uwp.edu) \\ (rileyd@uwp.edu)
}

With the continuous advancement of mobile technologies, sensing technologies and cloud computing, mobile technology services have unleashed immense opportunities in the field of healthcare. The motivation behind this paper is to focus on the existing mobile healthcare technologies and their impact on individual and community welfare. Sensing technologies and cloud computing are being increasingly used to enhance healthcare services and to reduce healthcare expenses. The evolving mobile healthcare technologies are confronting impediments regarding battery life, storage, bandwidth, security, scalability, reliability, and efficiency. In this paper, we present an overview of existing mobile healthcare applications and present a unified architecture for healthcare applications. In addition, we analyze the challenges of wireless sensor networks in the context of healthcare applications, and discuss future research directions of mobile healthcare technologies.

Keywords: Mobile Health, Home Healthcare, Interoperability, Personalized Monitoring, Self-Management, Scalability

\section{Introduction}

Mobile health is a new area of scientific development which has unleashed vast opportunities to improve patients' health and well-being. Mobile health offers realtime sensing of changes in health status and human behaviors; it allows fast analysis of health conditions, facilitates the adoption of remote healthcare services and helps to maintain a healthy lifestyle (Kumar et al., 2013). In today's world, mobile devices are among the most efficient and useful tools to communicate with people beyond space and time constraints. Smartphone and tablet PCs are getting increasing adherence due to their capability to facilitate rich user experience and numerous services via mobile applications that run on the devices or distant servers using wireless networks and cloud computing. Mobile devices are becoming an integral part of human life and the rapid development of mobile technology unwraps massive opportunities to improve healthcare systems (Dinh et al., 2013).

Currently, chronic diseases like diabetes, heart disease, blood pressure etc. are becoming more prominent and sometimes require lifelong medical care (Dillon and Znati, 2012). A large population of the elderly people are suffering from chronic diseases and visiting clinics for regular checkup and diagnosis (Hill et al., 2014) (Dillon and Znati, 2012). Also people who are living in remote areas are deprived of getting quality health care services due to lack of healthcare facilities, unavailability 
of quality health care professionals, lack of diagnostic tools (Ramesh et al., 2012). Mobile healthcare has the ability to facilitate better healthcare services to all these section of people either living in the rural area or having chronic disease and in need of continuous monitoring (Hill et al., 2014) (Ramesh et al., 2012) (Dillon and Znati, 2012). Mobile healthcare offers immediate monitoring, detection of variations in health status and enables the application of interventions, such as patient self-care and providing remote healthcare services (Kumar et al., 2013) (Sahithi et al.). A person's health status and behavior patterns can easily be tracked using sensors embedded in mobile devices or wearable on-body sensors (Kumar et al., 2013). Realtime health signals are collected from these sensors, processed and the results are sent to the healthcare providers for making patient's health related decisions (Ramesh et al., 2012). Recent study shows that healthcare spending is increasing day by day and mobile healthcare has the ability to provide efficient, innovative and lowcost way of services (Dillon and Znati, 2012). According to the World Health Organization (WHO), there are several global initiatives to engage mobile devices in healthcare, including health call centers, appointment reminders, mobile telemedicine and patient monitoring (Dirin et al., 2015).

Although mobile healthcare system improves the quality of healthcare, it also generates several challenges. These challenges are intensified by the resource scarcity that is integral with wireless sensor network platforms (Ko et al., 2010). Emerging mobile healthcare technologies are facing obstacles relating to signal quality, bandwidth, security, scalability, reliability and efficacy (Dinh et al., 2013) (Ko et al., 2010). In addition, mobile healthcare is a new emerging technology, concepts are unproven and sensing technologies in mobile devices have limitations.

Our paper presents a survey on existing mobile technologies in healthcare. The purpose of this paper is to provide readers an overview of different mobile technologies, contemporary in healthcare sector, including approaches and architecture. Our paper gives a survey of mobile technologies in healthcare, which help other researchers to have a quick overview about mobile technologies in healthcare industry. We analyze the approaches presented by the other researchers and propose our own architecture on integrated diabetes self-management system.

The rest of this paper is organized as follows. Section 2 of the paper presents an overview on the mobile health using sensing technology. Section 3 discusses mobile healthcare applications from the perspective of healthcare providers. Section 4 provides brief overview about home based mobile healthcare. In section 5, we propose an architecture for mobile healthcare applications. Section 6 discusses about mobile healthcare challenges and future research directions.

\section{An Overview of Mobile Healthcare Architecture}

\section{A. Architecture of Mobile Health Using Sensing Technology}

User friendly wireless devices, such as mobile phones, offer not only mobile communication, but also sensing, diagnosis, visual features and access to cloud. A person's health status and behavior patterns can easily be tracked using sensors embedded in a mobile phone or wearable on-body sensors. Mobile health has the capability to use mobile devices as personal labs that constantly measure a person's physiology parameters, behavior, social context, and environmental exposure. Mobile health has the capability to accelerate health research and apprise the formulation of public health policies (Kumar et al., 2013). 
Variety of sensors are already being embedded in mobile phones using sophisticated set of rules in mobile health applications. Innovative health, wellness and fitness related applications are being developed with the help of accelerometers and GPS sensing technology (Kumar et al., 2013). There are three main categories of sensors

- Movement sensors - are able to measure the rotation force in three axis environment. Accelerometers, gravitation and rotation sensors are movement sensor (Cimler et al., 2014).

- Environment sensors - measures environment conditions such as temperature, pressure, light intensity or moisture. Barometers, photometers and thermometers are environment sensor.

- Position sensors - monitors the physical position of the device by orientation sensors and magnetometers(Ko et al., 2010).

Android sensor framework contains several classes and interfaces to access sensors and gather raw data (Cimler et al., 2014). Some of these tasks are

- Determining availability of sensors (Cimler et al., 2014).

- Collecting basic information about sensors such as maximum range of sensors, manufacturer, category, energy consumption and resolution (Cimler et al., 2014).

- Retrieving raw sensor data and defining minimal period of gathering these data (Czaja et al., 2013).

- Registration and unregister listener monitoring variations on the sensor (Ghose and Bhaumik., 2012).

Figure 1 shows an overview of mobile health system. Sensors collect personal health data such as Electrocardiography (ECG), body temperature, limb movement. The signal is captured at the Bluetooth receiver module of the mobile phone and sent to signal processing module. The various segments of the signal are checked to identify abnormality in the signal. If the result is not normal, then the different signal attributes like amplitude, duration of each of the segments are compared with the normal values to make inference about the individual. The real-time result is stored in the mobile phone database for personal inference. These inferences are then logged into the back-end server database to make health inferences at the aggregated level to inform health research, practice, delivery, and policy formulation (Kumar et al., 2013).

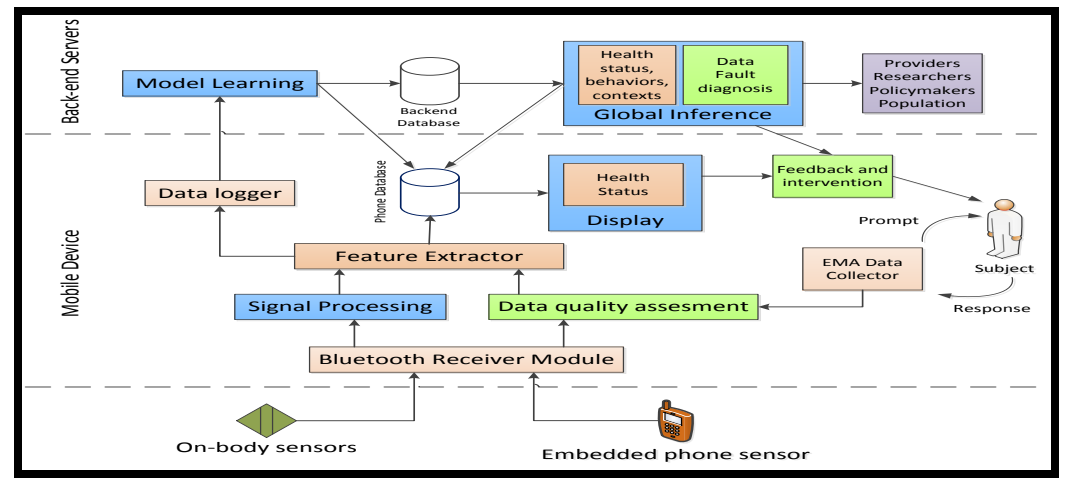

Figure 1 An Overview of Mobile Health System 


\section{B. Decision Support System}

Computer assisted diagnostic systems, also known as decision support systems, are widely accepted in health care and deal with monitoring patient health remotely by using wearable sensors (Ramesh et al., 2012). The architecture supports data collection and processing from multiple wearable wireless sensors for a variety of diseases like cardiac disease, pressure (Ramesh et al., 2012). The results are stored and sent to the healthcare professionals via Short Message Service (SMS) (Ramesh et al., 2012).

Figure 2 shows an overview of the decision support system. The key component of the system is a distant server providing clinical decision support. The body signal collected by the sensor unit is transmitted to the server through internet. The patients are constantly monitored and the medical signals are transferred anonymously. Each patient is assigned an identification number and identified by the unique number. The server receives different patient's data and upon receiving the data, the data is stored into the patient's database. The data is then scheduled according to their priority by means of a scheduling algorithm and retrieved from the server database for further analysis by the signal processing module.

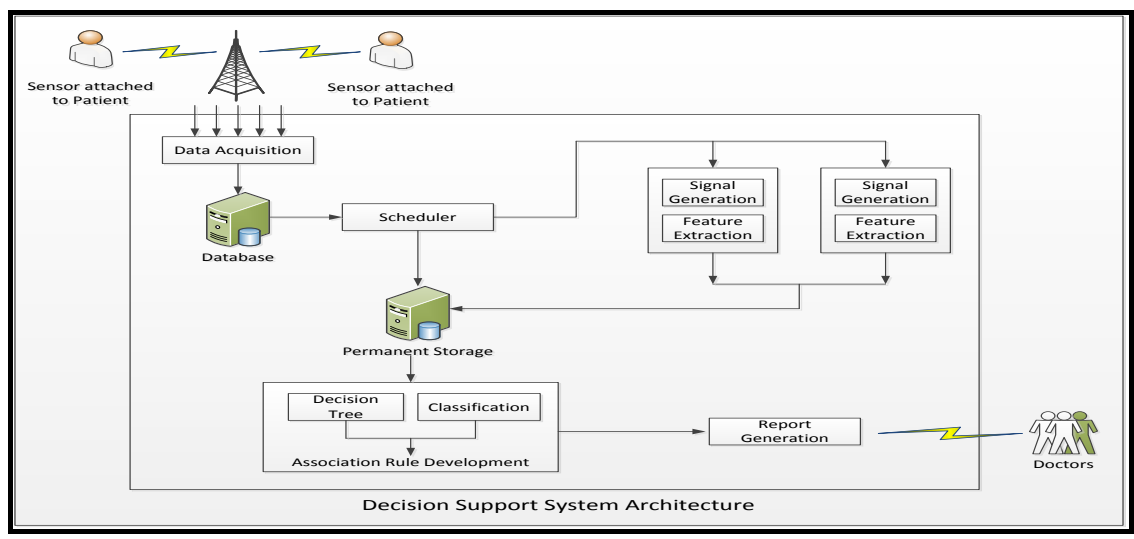

Figure 2 Decision Support System Architecture

Priority is assigned to the patient data depending on various risk factors such as hypertension, hypercholesterolemia, diabetes mellitus and previous heart attack or stroke so that the critical patient's data is analyzed first.

The signal processing module processes the signal by means of a number of signal processing algorithms depending on the type of the medical signal to extracts the various segments of the signal. The result of the analysis is sent to the physicians via messages to take the final decision regarding patient's condition (Ramesh et al., 2012).

The decision support system comprises of various modules as mentioned below (Ramesh et al., 2012).

1. Data Acquisition Module - Data is collected at frequent interval based on priority of patient's condition and stored in a database for further processing (Ramesh et al., 2012). 
2. Database Module - Consists of patient database, scheduler and a permanent storage. The data stored in the database are scheduled to be processed by the scheduler. Different sensors like ECG, Electroencephalogram (EEG) collect different variety of data and scheduler decides which data to be processed first. When multiple patient data is available in the global ready queue, the scheduling algorithm is invoked to decide the execution order based on their increasing order of the health risk and the time duration that they are in the queue. Inbound data, classified as high priority, is moved to the high priority queue and scheduled to be processed is scheduler using prioritized round robin algorithm. All other data are given to the low priority queue and have to wait in the low priority queue for a particular time frame. Once the waiting time is over the data is moved to the high priority queue for processing (Ramesh et al., 2012).

3. Signal Processing Module - Having various data processing algorithm, is capable of processing different types of medical data and provides reliable diagnosis results at early stage. The data fed by the scheduler to the signal processing module are regenerated to extract the various segments of the signal to check whether the signal is normal or not.

If the result is not normal, then the amplitude and duration of each of the segments are compared with the normal values to check for the presence of diseases (Ramesh et al., 2012).

4. Decision Module - the signal segment values are used as input to the classification algorithms to diagnose various types of disease. The technique also detects whether the signal is normal or abnormal. Decision trees are used as classification algorithm (Ramesh et al., 2012).

The anticipated decision support system has the capability to increase the efficiency, enhance health care quality and reduce health care costs. The system can also be used for early detection and notification for the forthcoming diseases. Notably, redundant variation in clinical practices can also be reduced (Ramesh et al., 2012).

\section{Concept of Tetherless Patients}

Tetherless patient is an ambulatory patient under continuous observation beyond the confines of traditional points of care (Dillon and Znati, 2012). The patient is monitored in a pervasive and ubiquitous way and capable of continuous communication with the caregivers (Dillon and Znati, 2012).

The solution is an expansion of contemporary healthcare delivery for a large and potentially growing segment of conditions and diseases like chronic disease that allows uninterrupted monitoring of patients beyond the traditional way (Dillon and Znati, 2012). To address the complex challenges of supporting this model, the solution focuses on a context-aware, mobile architecture that considers the entire route from sensor to provider (Dillon and Znati, 2012).

Figure 3 shows an overview of the system. One or multiple sensors attached to the patient body or embedded in patient's environment detect and communicate vital sign data to a central node, such as a smartphone or similar mobile device, which is termed as the coordinator (Dillon and Znati, 2012). 
The system evaluates the health data in the circumstance of the patient's current environment to detect potential problems, irregularities and variances.

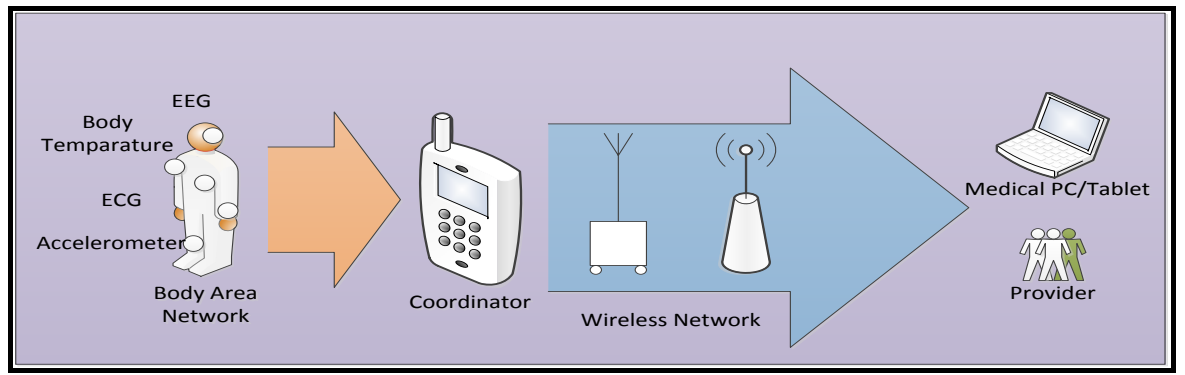

Figure 3 Infrastructure of Tetherless Patients

Patient's health data is registered into remote server storage and alerts are sent to healthcare provider using the existing provider networks (Dillon and Znati, 2012).

A number of solutions have been proposed regarding the tetherless care objective and a survey is presented in (Ko et al., 2010). All these solutions mainly propose different locations where information is gathered and processed (Ko et al., 2010). In the architecture, some portion of the analysis is moved to patient's mobile device and limits the broadcast of raw samples to the local area surrounding the patient using low power wireless communication technologies (Dillon and Znati, 2012). The solution also considers the patient's mobility by storing patient data on the smartphone while disconnected from wireless network, but doesn't account for patient's actions and criticality during the period of disconnection (Dillon and Znati, 2012). The major components of the implementation are coordinator and remote services.

a. Coordinator : Figure 4 illustrates the architecture for the coordinator. Coordinator comprises of three abstractions - virtual sensors, state descriptors and actions (Dillon and Znati, 2012). These are lightly attached objects and the computed results of each is accessible through a broadcast mechanism within the application (Dillon and Znati, 2012). To demonstrate these, assumption is made that a patient is wearing an ECG sensor and carrying an android smartphone with enabled location services (Dillon and Znati, 2012).

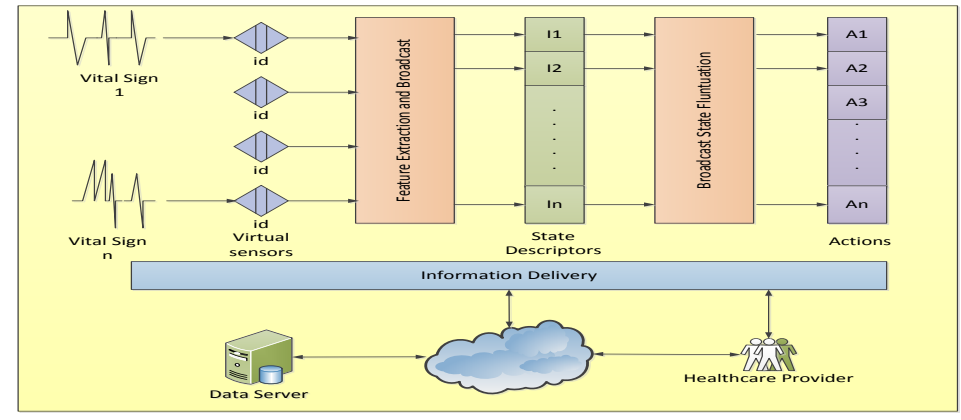

Figure 4 Architecture of Coordinator 
1. Virtual Sensors - Perform Two Main Functions

- Isolate the communication details and control one or more sources of data from the rest of the architecture (Dillon and Znati, 2012).

- Extract meaningful features from that data, which are broadcast to other objects (Dillon and Znati, 2012).

For example, an ECG virtual sensor receives ECG signal samples from patient's ECG device and stores the history of these samples for several seconds. The history is analyzed to locate the QRS complex, which is the combination of three graphical deflections on a typical ECG, and then initiate a broadcast based on the samples in that event (Dillon and Znati, 2012).

2. State Descriptors - collection of state descriptors perform the following activities collectively.

- Analyze and interpret the related aspects of the patient's context from specific virtual sensors (Dillon and Znati, 2012).

- Broadcast state fluctuations based on the observations made (Dillon and Znati, 2012).

For example, a heart-attack state descriptor has algorithms to evaluate the QRS complex for irregularities, and compute a probability of an oncoming heart attack (Dillon and Znati, 2012).

3. Actions - based on related changes, a set of actions implement the functionalities to be initiated. Each action depends on state descriptors and declares its associated state change based on updates from state descriptors (Dillon and Znati, 2012).

b. Remote Services: The web server is currently providing only data logging functionality and accepts messages from the coordinator and performs the necessary database processing to store the data in a database.

c. User Verification using Gait Recognition: Healthcare systems are susceptible to user spoofing, in which an adversary distributes his assigned device to other users and data collected from other users can be claimed as his own to procure more healthcare benefits (Ren et al., 2013). Most smartphones offer secret personal identification number as user verification method and verification based on personal identification number is not sufficient to mitigate spoofing (Ren et al., 2013).

Users' unique physical traits such as limb movement, body mechanics are hard to counterfeit and can be used to mitigate user spoofing in mobile healthcare systems (Ren et al., 2013). Gait analysis is an organized study of variations in limb movement, activity of the muscles and body mechanics (Ren et al., 2013). Human gait is a person's unique pattern of walking and can be used as unique discriminator (Ren et al., 2013). The presence of user spoofing is recognized by newly identified gait patterns which are different from a user's normal gait patterns. And the change in gait pattern is used to detect the attacks (Ren et al., 2013). Gait pattern can be used 
to prevent user spoofing in mobile healthcare system and the framework involves accelerometer embedded smartphones (Ren et al., 2013).

The adversary model provides an overview of the verification framework. Figure 5 shows the overview of the system. The framework can be implemented in two ways: server-centric and user-centric (Ren et al., 2013).

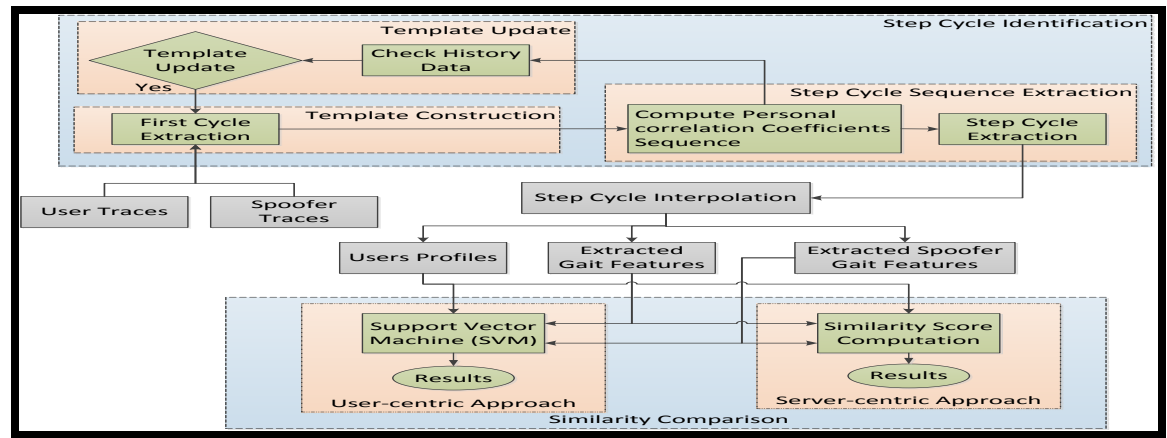

Figure 5 An Overview of Our User Verification System Using Gait Patterns

User verification is performed on the mobile device in case of user-centric approach. Initially user's profile is created and stored in the mobile device (Ren et al., 2013). User verification is then performed with low computational complexity (Ren et al., 2013). Correlation coefficient based approach is used to help computing similarity scores between the user profile and gait features that are extracted from user's mobile device (Ren et al., 2013). If the user verification fails, user spoofing is detected and the sensing data collected will not be reported back to the server (Ren et al., 2013).

Gait features that are extracted from accelerometer readings are sent to a centralized server for user verification in the server-centric approach (Ren et al., 2013). Data mining based technique is used to perform user verification by comparing the stored user profiles with gait features extracted from user's mobile device (Ren et al., 2013). The server will decide whether to accept the sensing data collected from user based on the user verification result (Ren et al., 2013).

The system can be deployed in both user-centric as well as server-centric framework (Ren et al., 2013). The gait pattern based user verification system comprises of three components: Step cycle identification, step cycle interpolation and similarity comparison (Ren et al., 2013).

1. Step cycle identification: Gait pattern is repeatable, a template is used to recognize the first distinguishable step cycle (Ren et al., 2013).

2. Step cycle interpolation: The number of samples in step cycle depends on the inconsistent walking speed of the user (Ren et al., 2013).

3. Similarity comparison: The walking speed of a user fluctuates but the distinct feature is implanted in each gait pattern remains unchanged (Ren et al., 2013).

\section{Mobile Healthcare Based on Healthcare Providers}

\section{A. Enabling Secure Mobile Access Using Generic Bootstrapping Architecture}

Medical applications are already being integrated into mobile devices such as smart phones, PDAs and used to retrieve patient's electronic health data. The architecture 
deals with the secure transfer of individual medical data using Generic Bootstrapping Architecture (GBA), in 3G mobile network (Shanmugam et al., 2006). Appropriate security measures which include authentication, authorization, integrity, access control and privacy safeguards are considered to protect the privacy of medical information (Shanmugam et al., 2006).

The GBA implements the mutual authentication between the users and network applications (Shanmugam et al., 2006). GBA authenticates user, based on the long term credentials present in the Subscriber Identity Module (SIM). The security of GBA lies in the assumption that the access to that long term shared secret is difficult and hard to break (Shanmugam et al., 2006).

Figure 6 shows GBA reference model which includes the client User Agent (UA), Application Function (AF), Bootstrapping Function (BF) and Home Subscriber Systems (HSS) communicating through appropriate reference points. The following sequence of actions will take place to perform the bootstrapping (Shanmugam et al., 2006).

1. Based on UA's requests to $\mathrm{AF}, \mathrm{AF}$ notifies the user to perform authentication with the BF (Shanmugam et al., 2006).

2. UA contacts the BF and BF retrieves the credentials from the HSS to complete the authentication process (Shanmugam et al., 2006).

3. UA and BF mutually authenticate using the specified authentication and key agreement protocol. Then application specific shared key is derived (Shanmugam et al., 2006).

4. AF interacts with BF, gets the shared key from BF and authenticates the user, when requested (Shanmugam et al., 2006).

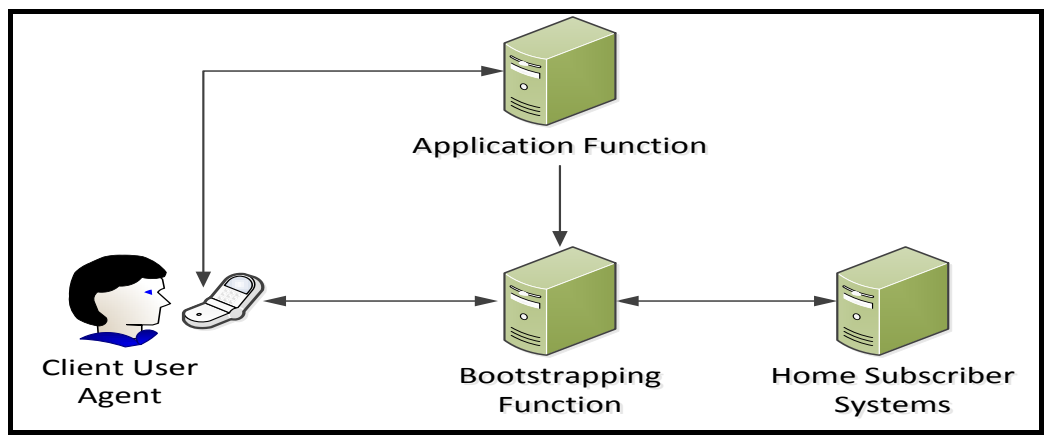

Figure 6 GBA Reference Model

Figure 7 shows the high level electronic health care architecture and explains the relationship between the participating entities. There are four logical components as described below (Shanmugam et al., 2006).

1. User - requests medical records to be sent to a particular hospital (Shanmugam et al., 2006).

2. Network operator - facilitates for accessing the service and provides identity for the user (Shanmugam et al., 2006).

3. Service provider - provides the service of managing user's medical records (Shanmugam et al., 2006). 
4. Hospital - requires the medical records in order to diagnose user (Shanmugam et al., 2006).

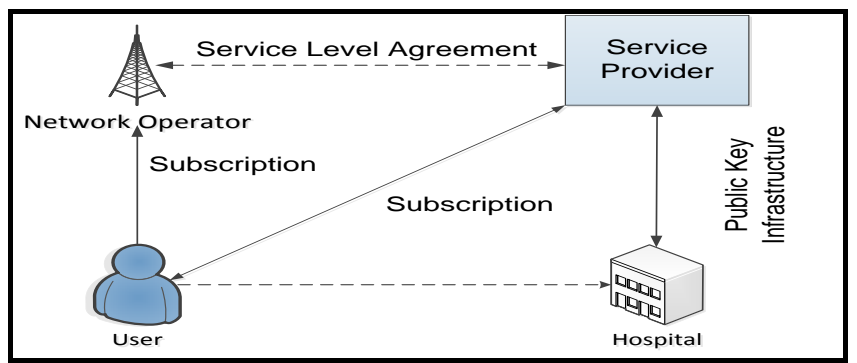

Figure 7 Architecture for Accessing Electronic Health Care Records

\section{B. Wireless Distributed Framework}

Wireless Electronic Healthcare Record (W-EHR) is a wireless distributed architecture that enables mobile users to access electronic medical records in a secure and transparent way (Belsis et al., 2009). Security management of devices with different roles within this framework is achieved using a hierarchical policy based approach (Belsis et al., 2009). All the transactions are encoded using a hybrid approach that utilizes symmetric and asymmetric encryption techniques (Belsis et al., 2009). The encryption technique enables secure communication of information without excessive processing resources in the mobile devices, having limited capabilities (Belsis et al., 2009). The architecture facilitates authorized personnel to access patient information in an accurate and secure manner within a wireless LAN (Belsis et al., 2009).

The hierarchical policy based approach distinguishes two roles between the mobile nodes (Belsis et al., 2009). Manager Nodes (MNs) are devices held by doctors and are responsible to perform more advanced operations (Belsis et al., 2009). The other one is Terminal Nodes (TNs) which allow to perform simple operations and usually held by support medical personnel (Belsis et al., 2009).

The architecture allows secure distribution of medical records and guarantees interoperability and scalability. Interoperability is the communication between information technology system and software application. Scalability is the ability of a system to handle increasing amount of work (Belsis et al., 2009).

In a healthcare organization, each user has a specific role with definite set of security permissions and uses devices with different processing capabilities (Belsis et al., 2009). A hierarchical approach is adopted to distinguish three types of network nodes (Belsis et al., 2009).

1. Central Nodes (Cns) - stores the medical database and are responsible for authentication and access control enforcement. CNs are also responsible for the operation of the policy management module and maintain the medical database (Belsis et al., 2009).

2. Manager Nodes (MNs) - assigned with more advanced tasks. Local Policy Decision Point (PDP) and Policy Enforcement Point (PEP) module are installed in MNs. MNs allow enforcement of local policies, enabling access to other doctor's to the device's local repository (Belsis et al., 2009). 
3. Terminal Nodes (TNs) - lack in respect to MNs in resources and are assigned secondary tasks. TNs perform simple operations, such as notifying nurse about an emergency or providing details about a patient's prescription and schedule. TNs are not allowed to access sensitive medical data (Belsis et al., 2009).

Figure 8 shows the proposed distributed W-EHR architecture. Both TN and MN nodes are able to recognize whether they reside within the clinic network or in an unknown environment, with the aid of signed messages identifiable by each (Belsis et al., 2009). The system avoids unauthorized communication to or from the device when resides outside the network (Belsis et al., 2009). The distributed policy authorization module is comprehended by means of object-oriented software architecture, using Java (Belsis et al., 2009).

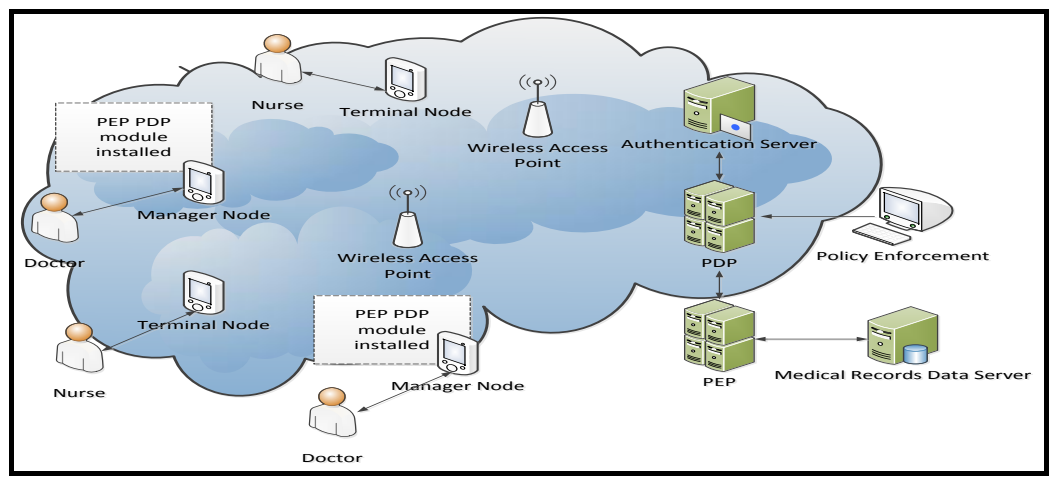

Figure 8 Distributed W-EHR Architecture

\section{Home Based Mobile Healthcare}

\section{A. Diabetes Self-Management Using Diamond}

Diabetes is one of the prominent chronic disease and often requires lifestyle adjustment and sometime lifelong medical care. Evidence suggests that technologyfacilitated telemedicine can help patients to improve their self-management and their relationship with healthcare professionals (Hill et al., 2014). These technologies can be considered as Self-Service Technologies (SSTs), enable patients to produce services with little involvement from healthcare professionals (Hill et al., 2014).

Figure 9 shows the high level architecture of diamond model. Diamond is a SST which facilitates diabetes monitoring capabilities to diabetes patients and healthcare professionals. Diamond is easy to use and requires diabetes patients to enter their glucose HA1C readings systematically using simple user interface before sending them for processing to the medical facility. Diamond operates like Short Message Service (SMS). Patient requires a mobile handset and data plan to communicate with the provider. The anticipation is that healthcare provider needs to upgrade software in the existing environment without any upgrade to computer hardware. Interaction frequency between the patients and providers is expected to be high, though depends on the treatment procedure and prescription from the provider. Interaction frequency is not only a measure of cost effectiveness but also a way of enhancing patient's satisfaction over traditional clinical-patient interaction. The easy, economical and 
pervasive solution enables healthcare professionals to closely monitor diabetes trends and interact with patients easily (Hill et al., 2014).

\section{B. Long-distance Healthcare for Elderly People}

Several governments around the world are promoting program like tele-health for elderly people with the following capabilities (Shih et al., 2013).

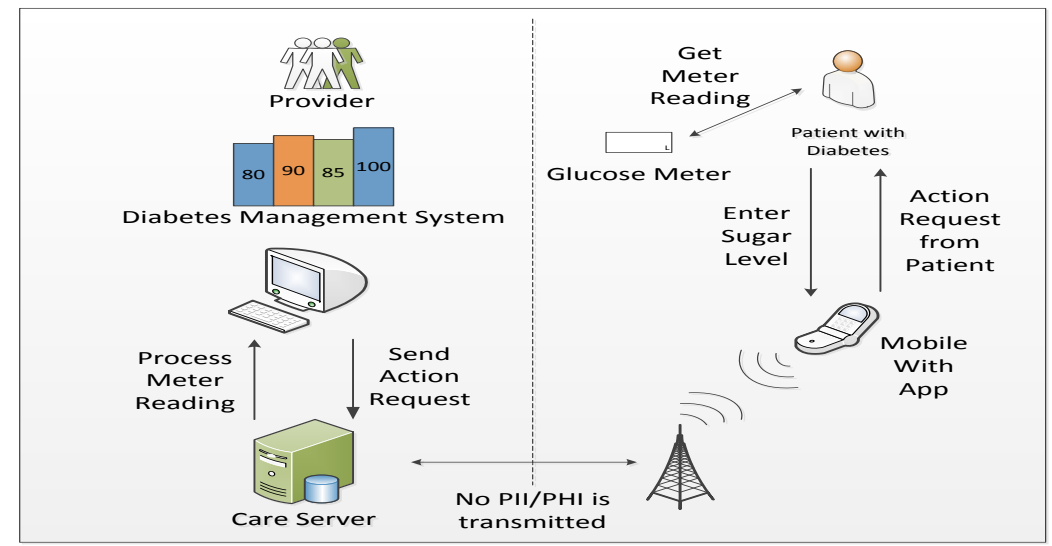

Figure 9 High Level Architecture of Diamond Model

1. Measurement - patient is able to provide their own physical data using health care devices like sensor, app or internet (Shih et al., 2013).

2. Communicative - patient is able to contact medical professionals to get answer for any questions using the system (Shih et al., 2013).

3. Interactive - patient is able to check their history of physical changes or get immediate medical care in case of emergency or any need (Shih et al., 2013).

4. Educational - patient is able to get health care related information using tele-healthcare system (Shih et al., 2013).

Continuous evolution of information technology is changing the way of providing health care facilities both in home and outside of home. Both wearable sensors and mobile embedded sensors are being widely used to measure patient's vital sign and activity by non-invasive methods (Shih et al., 2013). Sensors are able to detect signal such as EEG, ECG, pulse, temperature, gait, blood glucose and limb movement (Shih et al., 2013).

The prototype system is divided into two parts, the user and monitoring client. Figure 9 shows an overview of the system. Activity sensor, fixed to the body using straps or embedded in mobile device, is used to detect signal and the signal is sent to the smartphone through Bluetooth interface. The sensor is able to detect signals like heart rate, respiratory rate, body temperature, posture, and limb movement etc. The application code is written in Java and uses SQL lite databases to store sensor data using Android platform. Finally the data is sent to server through Wi-Fi or $3 \mathrm{G}$ signal. For each different types of body signal threshold limit is set and the system triggers the warning procedure if the sensed data goes above the pre-set limit (Shih et al., 2013). 


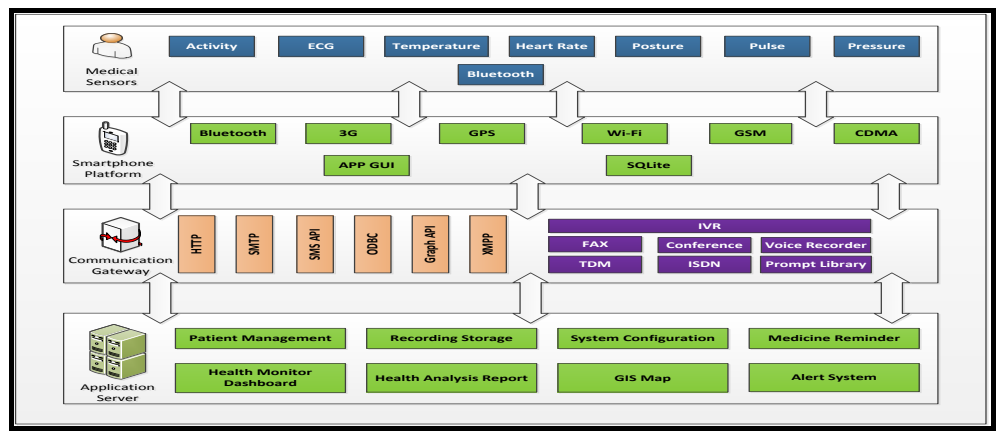

Figure 10 System Diagram

The server side application is responsible for user management, like facilitating medication reminders and comprehensive warning function (Shih et al., 2013).

\section{Proposed Architecture}

There are over 25.8 million diabetic patients, including children and adult in USA, according to new 2011 prevalence data estimates from the Centers for Disease Control and Prevention (CDC, 2011). As a chronic disease, diabetes patients require ongoing medical attention and engagement in self-management education and support to reduce the risk of long term disability and complications (ADA, 2012). Self-management procedures are naturally data intensive and require the collection, storage, and analysis of large amount of data in a systematic way.

Recent advances in mobile technologies enable mobile devices to serve as the patient terminals in telemedicine conditions and allow data services including diabetes self-management. In our paper, we propose a mobile health architecture of integrated diabetes self-management system. The architecture has the following functionalities: manual \& automated data-entry through the use of wireless sensors, a decision support component that provides personalized evidence-based recommendations, advanced statistical techniques for diabetes modeling and prediction.

Figure 11 shows the overview of the proposed mobile health architecture. The proposed model comprises two main modules.

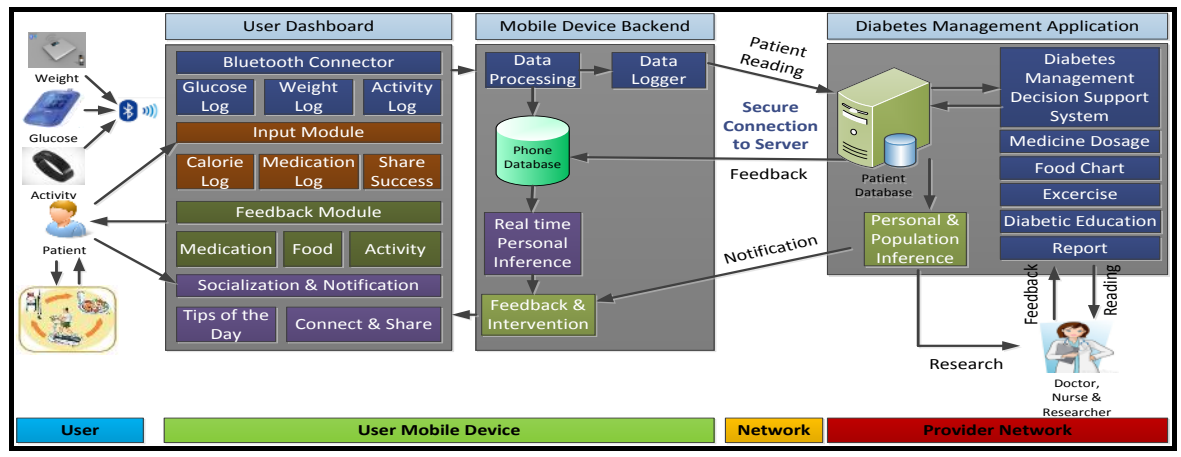

Figure 11 Integrated Diabetes Self-Management System 


\section{A. User Mobile Device (e.g. Smart phones)}

B. Provider Web-Based Diabetes Management Application.

These two modules are linked by an existing mobile networks (4G/LTE) and/or Wi-Fi networks linked to the core IP network.

\section{A. User Mobile Device}

The application runs on the patient's mobile device to perform the following tasks

1. Collects the daily blood glucose measurements, weight and activity/calorie burned from the patient glucose meter, weighing machine and activity tracker devices respectively using Bluetooth connectivity.

2. Patient enters the diet and medication details using the mobile application dashboard.

3. Patient's vital data are captured in the data processing module of the mobile device.

4. Patient's vital data are stored in the mobile phone database for personal view.

5. The data logger captures all the vital data and sends the details to provider server using secure connection.

6. The mobile device stores all the feedback from the provider into the phone database for user view. Feedback includes the medication details, dietary and activity details recommended for the patient.

7. Notifications such as tips of the day, success story of other patients, medication \& blood glucose reading reminders are also captured from provider and displayed in the mobile dashboard.

8. Patient can also see all the logs for certain period of time and is able to share own success story for diabetic community.

\section{B. Provider Web-Based Diabetes Management Application}

The application is hosted by a web health server which is accessible by health care professionals and the patients.

1. Patient data from mobile device is first stored in the server database and then fed to a decision support system.

2. Decision support system has several data processing algorithms and is capable of processing different types of medical data and provides reliable diagnosis results at early stage to the physicians.

3. The result of the diagnosis is then checked by the physicians to take the final decision regarding patient's medication, dietary and activity details.

4. These personal inferences are then logged into the server database to make health inferences at the community level to inform health research and policy formulation.

In our architecture we proposed the server-centric approach, where core data and applications reside on the server, as the main decision support system. But the system can be designed in both user-centric as well as server-centric frameworks. Our paper proposed the model architecture with the aim of shifting the emphasis from a traditional clinician centric approach to a patient centric approach in an effort to support diabetes self-management. The proposed model can be easily integrated to 
an existing electronic health platform which offers distant manageability capabilities and provides telemedicine services.

\section{Discussion and Future Work}

There will be around 72 million people aged above 65 by 2030 in USA (Czaja et al., 2013). Healthcare for elderly people is gradually getting more attention not only in USA but also rest of the world. Majority of the elderly people are suffering from chronic diseases and they end up visiting hospitals and clinics for emergency care, regular checkups, procedures, and diagnosis (Shih et al., 2013). Circumstantial evidence suggests that acceptability of the wireless technology is increasing to facilitate chronic disease self-management. Computing and sensing technologies are creating new opportunities to change the way healthcare is delivered now. Chronic conditions could be easily treated in an outpatient manner and receive comparable quality of care with lesser cost (Shih et al., 2013).

The literature on technology-based disease self-management and its impact on healthcare quality is limited. A number of dimensions for evaluating mobile technologies in healthcare context, based on how consumers utilize and differentiate a service, have been proposed. Ease of use is one of the most prevailing outcomes in the technology adoption model; it can be described as the degree to which a person believes that using a particular technology is free of effort (Davis, 1989). Ease of use not only reduces patient's effort, but also reduces social risks. Speed of delivery is essential in any technology-driven model and expected to provide faster services than face-to-face services. Assurance refers to confidentiality, security and retrieval aspects when services fail. Protecting customer privacy is one of the key elements of technology based self-management model. Also perceived control of consumers over service processes needs to be satisfactory (Hill et al., 2014).

Although mobile healthcare has several advantages, there are numerous challenges which need to be addressed. The integration of multiple platforms like sensors, mobile devices, cloud, mobile networks and provider networks creates several technical challenges. This section lists several research issues in mobile healthcare.

Data collection using mobile health sensors introduces numerous data-quality challenges (Kumar et al., 2013). Sensor measurement is sometimes improper due to incorrect placement of sensors, attachment errors, variability inherent in patient's daily activities and the mobile environment (Kumar et al., 2013). Signal quality from these sensors are often missing clinical relevance and incompatible to offer conclusive analysis for complex health conditions. The main challenge lies in designing smaller size, cost effective sensors which are compatible with smartphone and able to perform various tests that can only be done in a clinical setting. Bio-molecular sensing, imaging and bioelectric sensing are precisely important and research is being performed to integrate these sensors in mobile devices.

In applications such as gait recognition, accelerometer readings pose several challenges. First, the quality of accelerometers embedded in smartphones rarely have high standards and generate lower sampling rate. Recording of unique gait pattern can be difficult by using lower sampling rates. Second, during the verification process, user's gait pattern varies and creates difficulties to recognize the step cycles properly. Third, the user verification process is expected to be able to complete with small number of measurements. Last, a malicious user of the system can imitate a 
user's walking pattern to deceive the system although a person's walking pattern is hard to break (Ren et al., 2013).

Bandwidth limitation is another concern due to the growing number of mobile and cloud users (Dinh et al., 2013). Fourth generation (4G) networks increase the bandwidth capacity for subscribers that helps to overcome the bandwidth limitation. Service availability is another issue in mobile network driven services. Users are not able to get service due to traffic congestion, network failures and out-of-reach signals. Location information is often not available especially at indoor facilities. At times, applications are prohibited from uploading the current location. To overcome this, the system is supposed to allow users to enter the location manually and use geocoding API available from Google maps to arrive at a coarse location coordinates for the place (Ghose et al., 2012).

Energy is another real challenge for mobile health (Kumar et al., 2013). When mobile phones are engaged in continuous sensing, lifetime of a mobile phone is considerably compromised. Privacy is another major concern in healthcare sector and is managed by Health Insurance Portability and Accountability Act, 1996 (HIPAA). The main objective of HIPAA is to ensure that individual health related information remains private and secure. Mobile health systems pose immense challenges for HIPPA. One challenge is attributed by the openness of mobile health systems. Health information is transferred, owned and operated by multiple parties involved. Ensuring privacy and security are very difficult in such a setting. Also data collected from personal devices and sensors has contributed to an even bigger privacy challenge.

Thus mobile health systems need to focus more on user data security and privacy going beyond the traditional architecture. Also, the system must adhere to the available resources and communication framework such that patient's security is never compromised and, furthermore, the system must meet the security and privacy guidelines outlined by HIPAA (Dillon and Znati, 2012). Safety issues are important for mobile healthcare both from health and engineering perspectives. From health perspective, safety means correctness and quality of the information. From engineering perspective, safety means that devices used in mobile healthcare are not causing any harm or inconvenience to the user. Designers must note all of these factors while designing their products (Kumar et al., 2013).

Another challenge lies in providing services at least equivalent to traditional care. The system must guarantee uninterrupted monitoring of patient's vital signs and support the health related real-time reporting (Dhumal et al., 2014). In the developing world, community health workers (CHWs) are often the only connection to healthcare for millions of people. CHWs serve people by conducting, monitoring assessment exercises, disease surveillance and providing point-of-care diagnostic support (Liu et al., 2011). CHWs also work as liaison between the households in their communities and skilled healthcare practitioners in clinics and hospitals, for example, for the treatment of intricate illnesses or specialized care. Hence, supporting CHWs at the point-of-care is thus of important task. This can be achieved through mobile health platforms. Mobile health has the ability to improve CHW performance by enabling the capture, storage, transmission and retrieval of health data. Mobile health technologies can act as a convenient and cost effective way to save lives and improve care in the context of CHWs. (Gatara et al., 2014).

Some of the future directions for our work include expanding the use of mobile healthcare architectures to other facets of healthcare. For example, the 
pharmaceutical industry has its own challenges in terms of patent expirations, price pressures, and increased regulatory scrutiny (Chitra and Krishnan, 2007). The issue of price transparency is paramount in pharmaceutical industry. Mobile healthcare has the ability to provide patients with price transparency through apps devoted to comparing prices between different medication options and pharmacies. Another feature of mobile healthcare can enable patients to review and monitor adverse drug interactions between multiple medications; this is especially useful for patients with multiple comorbid conditions. Another direction for future work lies in the need for integrating healthcare data from mobile devices for discovering new patterns and treatment methods. For example, application of knowledge discovery to medical databases can yield new directions for treatment of diseases (Steiner et al, 2006) or even new approaches for reducing healthcare costs (Chalasani et al., 2014). Since data from mobile health devices is fragmented and widely distributed, it may be difficult to integrate myriad pieces of data to enable knowledge discovery. Architectures and methods that facilitate seamless integration of mobile healthcare data with traditional electronic health records will be a significant direction for future research.

\section{References}

1. Kumar, S., Nilsen, W., Pavel, M., \& Srivastava, M. (2013). Mobile health: revolutionizing healthcare through transdisciplinary research. Computer, (1), 2835.

2. Ren, Y., Chen, Y., Chuah, M. C., \& Yang, J. (2013, June). Smartphone based user verification leveraging gait recognition for mobile healthcare systems. In Sensor, Mesh and Ad Hoc Communications and Networks (SECON), 2013 10th Annual IEEE Communications Society Conference on (pp. 149-157). IEEE.

3. Shanmugam, M., Thiruvengadam, S., Khurat, A., \& Maglogiannis, I. (2006, November). Enabling secure mobile access for electronic health care applications. In Pervasive Health Conference and Workshops, 2006 (pp. 1-8). IEEE.

4. Belsis, P., Vassis, D., Gritzalis, S., \& Skourlas, C. (2009, June). W-ehr: a wireless distributed framework for secure dissemination of electronic healthcare records. In Systems, Signals and Image Processing, 2009. IWSSIP 2009. 16th International Conference on (pp. 1-4). IEEE.

5. Hill, S. R., Troshani, I., Goldberg, S., \& Wickramasinghe, N. (2014). Improving Healthcare Service Quality and Patients' Life Quality Through Mobile Technologies: The Case of Diabetes Self-management. In Lean Thinking for Healthcare (pp. 345-359). Springer New York.

6. Ramesh, M. V., Anu, T. A., \& Thirugnanam, H. (2012, September). An intelligent decision support system for enhancing an m-health application. In Wireless and Optical Communications Networks (WOCN), 2012 Ninth International Conference on (pp. 1-5). IEEE.

7. Dillon, P., \& Znati, T. (2012, October). Towards an architecture for mobile healthcare. In Local Computer Networks (LCN), 2012 IEEE 37th Conference on (pp. 260-263). IEEE.

8. Shih, C. C., Chou, K. Y., Keh, H. C., Cheng, Y. C., Yu, P. Y., \& Huang, N. C. (2013, September). Building Long-Distance Health Care Network Using Minimized Portable Sensors and Active Alert System. In Network-Based 
Information Systems (NBiS), 2013 16th International Conference on (pp. 401404). IEEE.

9. Sahithi, M. K., Muneer, M. M., \& Srinivasulu, M. C. (2015, January). SPOC: A Secure and Privacy-Preserving Opportunistic Computing Framework for Mobile Healthcare Emergency. International Journal of Computer Science information and Engineering Technologies (IJCSIET)

10. Dinh, H. T., Lee, C., Niyato, D., \& Wang, P. (2013). A survey of mobile cloud computing: architecture, applications, and approaches. Wireless communications and mobile computing, 13(18), 1587-1611.

11. Cimler, R., Matyska, J., \& Sobeslav, V. (2014, July). Cloud based solution for mobile healthcare application. In Proceedings of the 18th International Database Engineering \& Applications Symposium (pp. 298-301). ACM.

12. Ghose, A., Bhaumik, C., Das, D., \& Agrawal, A. K. (2012, June). Mobile healthcare infrastructure for home and small clinic. In Proceedings of the 2nd ACM international workshop on Pervasive Wireless Healthcare (pp. 15-20). ACM.

13. Dirin, M., Dirin, A., \& Laine, T. H. (2015, January). User-centered design of a context-aware nurse assistant (CANA) at Finnish elderly houses. In Proceedings of the 9th International Conference on Ubiquitous Information Management and Communication (p. 39). ACM.

14. Gatara, M., \& Cohen, J. F. (2014, September). Mobile-Health Tool Use and Community Health Worker Performance in the Kenyan Context: A TaskTechnology Fit Perspective. In Proceedings of the Southern African Institute for Computer Scientist and Information Technologists Annual Conference 2014 on SAICSIT 2014 Empowered by Technology (p. 229). ACM.

15. Ko, J., Lu, C., Srivastava, M. B., Stankovic, J., Terzis, A., \& Welsh, M. (2010). Wireless sensor networks for healthcare. Proceedings of the IEEE, 98(11), 1947-1960.

16. Czaja, S., Beach, S., Charness, N., \& Schulz, R. (2013). Older adults and the adoption of healthcare technology: Opportunities and challenges. In Technologies for active aging (pp. 27-46). Springer US.

17. Davis, F. D. (1989). Perceived usefulness, perceived ease of use, and user acceptance of information technology. MIS quarterly, 319-340.

18. Liu, A., Sullivan, S., Khan, M., Sachs, S., \& Singh, P. (2011). Community health workers in global health: scale and scalability. Mount Sinai Journal of Medicine: A Journal of Translational and Personalized Medicine, 78(3), 419435.

19. Centers for Disease Control and Prevention (CDC), \& Centers for Disease Control and Prevention (CDC). (2011). National diabetes fact sheet: national estimates and general information on diabetes and prediabetes in the United States, 2011. Atlanta, GA: US Department of Health and Human Services, Centers for Disease Control and Prevention, 201.

20. American Diabetes Association. (2012). Standards of medical care in diabetes-2012. Diabetes care, 35, S11.

21. Dhumal, P., Chalasani, S., and Wickramasinghe, N. (2015). Financial Model for Investment Recovery Period in Electronic Health Records Implementations. International Journal of Economics and Business Research. 9(1), pp. 65-79. 
22. Chalasani, S., Jain, P., and Dhumal, P., Moghimi, H., and Wickramasinghe, N. (2104). Content Architecture Applications in Healthcare. Health and Technology, 4(1), pp. 11-19.

23. Chitra, S., Krishnan, P. (2007, March). Evolving Operational Decision Models in the Pharmaceutical Industry. International Journal of Operations and Quantitative Management. Vol. 13, No. 1. March 2007.

24. Steiner, Maria, et al. (2006, March). The Influence of Exploratory Data Analysis on the KDD Process: An Application to Medical Diagnosis. International Journal of Operations and Quantitative Management. Vol. 12, No. 1. March 2007.

\section{About Our Authors}

Mukta Mukherjee completed her MS in Computer Information Systems from the University of Wisconsin-Parkside in 2015. She is currently working as a software engineer at the University of Wisconsin-Parkside's Technology department. As a software engineer, she is responsible for managing the Learning Management Systems. Her areas of interest include Healthcare Technologies, Mobile Technologies, and Business Analytics.

Suresh Chalasani is a professor in the Business department at UW-Parkside. Prior to joining UW-Parkside, he worked as an assistant professor at UW-Madison. He also served as a consultant for several companies in the Chicago-Milwaukee corridor. Dr. Chalasani's educational background includes a $\mathrm{PhD}$ from the University of Southern California. Dr. Chalasani's current research and teaching interests include management information systems, technologies for business supply chains, transforming business processes using technologies, sustainable management, healthcare management, and healthcare technologies. He is a senior member of IEEE and a member of the Association for Information Systems (AIS). He has received numerous research and teaching grants from the National Science Foundation, University of Wisconsin System, and WiSys Technology Foundation. Dr. Chalasani has published an extensive number of research articles in journals such as IEEE Transactions on Computers, IEEE Transactions on Parallel and Distributed Systems, IEEE Transactions on Communications, and Information Systems Frontiers. He has also presented papers at a number of IEEE- and AIS-sponsored academic conferences. He is on the editorial board of the International Journal of Biomedical Engineering and Technology, where he co-edited a special issue on "Nanotechnology for Medical and Surgical Applications." He also served as a guest editor for the IEEE System's journal's special issue on "RFID Technology: Opportunities and Challenges." Additionally, Prof. Chalasani serves on the international program committees for several conferences.

Derek Riley studied computer science and mathematics at Wartburg College in Waverly, IA. He earned his PhD in computer science from Vanderbilt University in 2009. The main focus of Derek's graduate school research was modeling, simulation, and computational analysis of biochemical processes using stochastic techniques. Currently Derek does research in modeling and software engineering and is a faculty member at the University of Wisconsin- Parkside. 